\title{
System for Aircraft Data Value Demonstration
}

\author{
Stanislas Martin \\ AIRBUS Operations SAS, 316 route de Bayonne 31060 Toulouse, France \\ stanislas.martin@airbus.com
}

\begin{abstract}
:
In the frame of the Skywise data avionics platform, an end-to-end prototype was developed, certified and deployed in airlines, with the objective to demonstrate the value of aircraft data.

This on-board connectivity system is composed of existing flight test means to acquire massive data on board and of standard industrial components to transmit data directly to Airbus data lakes after each landing via standard $4 G$ infrastructure.

These data were then analysed by a Smart Data Analytics plateau to develop algorithms, in order to improve maturity of the aircraft or give recommendations to better operate the aircraft.

Thanks to a strong commitment and a trans-functional and trans-national team work, it has been possible to design, manufacture and certify the Skywise-demonstrator in a very short time frame (five months).

The system has been installed on in-service aircraft by the team and is operating in real airline operations. Since its entry into service in December 2016, the demonstrator has sent over 10 Terabytes (TB) of in-service data from five airlines worldwide. More than 100 algorithms have been developed and validated by Airlines, thus demonstrating the value of aircraft data analysis.
\end{abstract}

Key words: Data, connectivity, value, prototype, certification.

\section{Context}

For several decades, aircraft include a high number of avionics systems which are exchanging data between each other. However, on many aircraft, most of these data remains "inside" the aircraft and is accessible neither by the airline nor by the manufacturer. This is particularly the case for the Single Aisle aircraft family (A319/A320/A321) which initial conception did not integrate the capability to retrieve a high amount of aircraft internal data. Indeed, standard systems allow retrieving a few hundreds of parameters amongst the 24,000 really present.

Yet, it was strongly foreseen that accessing to more in-service data could bring great benefits to all parties. It would indeed be a way to improve knowledge on how aircraft are operated all along their life and in their real commercial environment.

The assumption was therefore that analyzing more in-service aircraft data would lead to:

- Improving the design of the aircraft (in addition to the flight test data traditionally used for this purpose)

- Enhancing services and
recommendations to operate the
aircraft more efficiently

- Solving in-service issues quicker and/or more efficiently

While launching a long-term activity to develop a data collector and connectivity system that could be installed on the whole Single Aisle aircraft fleet, it was decided to quickly develop an in-service demonstrator to:

- Run this scenario on a few in-service aircraft in order to confirm these benefits. In other words, to demonstrate data value.

- De-risk the industrial solution, by better identifying the pain points and upcoming difficulties.

This in-service demonstrator was also the opportunity to develop partnership with some airlines by addressing some concrete use cases at an early stage, thus allowing more efficient work with common interest between Airbus and its clients.

This article will describe the chosen solution for the embedded part of this demonstrator. It will then explain how it has been implemented, focusing on the challenges encountered and the success key factors. Finally, general results will be given. 
It should be noted that it is not in the perimeter of this document to address legal and financial topics.

\section{Technical solution}

Considering a strong timing constraint, the opportunity to use already existing systems was essential. Consequently, the solution was built on systems already used on flight test aircraft, and completed with commercial-of-the-shelf (COTS) systems.

The on-board system consists in two main functions described below:

- Acquisition of on-board data

- Automatic wireless transmission from board to ground

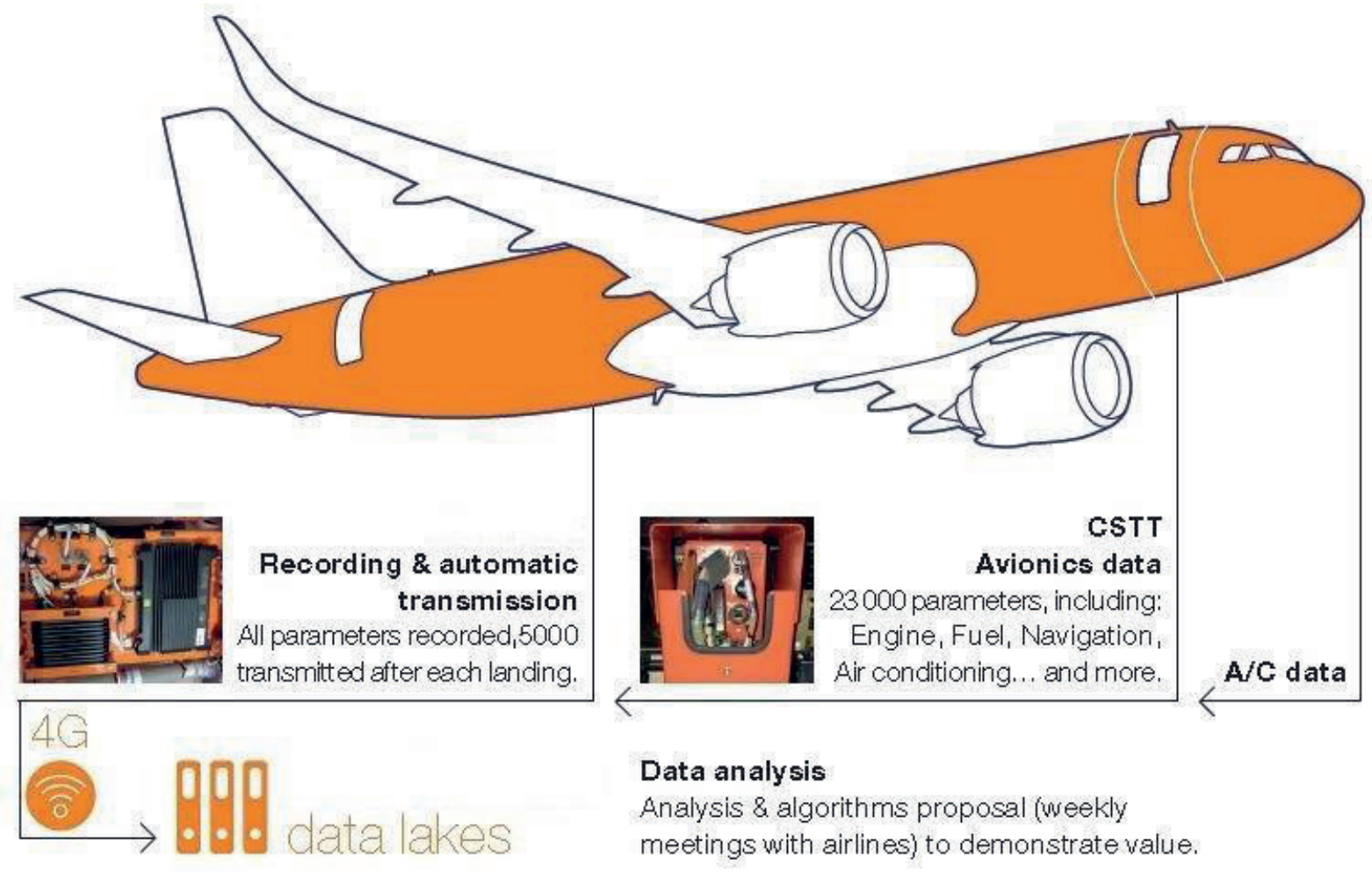

Fig. 1. General architecture principle of the demonstrator

The first function - acquisition of on-board data - is ensured by an acquisition unit already largely used for the flight tests of the production aircraft. This system is called PATS (Production Aircraft Test System) for its use in a flight test context, and is called CSTT (Customer Support Test Tool) in its certified version. It consists in a 4-MCU ARINC-600 chassis which can easily be installed in the electronic bay (in identified available slots) and connected to the aircraft avionics ARINC-429 buses. The CSTT system then automatically and continuously receives and acquires the complete avionics data: every sample of every label on all the buses, which corresponds to a set of approximately 24,000 parameters. The system then merges those parameters on a single Ethernet wire.

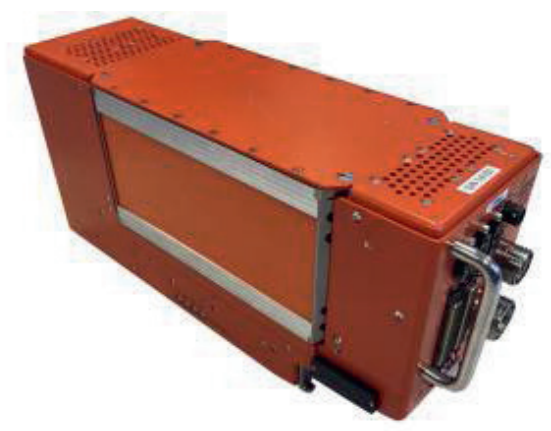

Fig. 2. Acquisition unit (CSTT system)

The second function - Automatic wireless transmission from board to ground - is ensured by a connectivity system built around COTS components (an industrial $4 \mathrm{G}$ router and its antenna, and ruggedized computing unit) associated with $\mathrm{FTI}$ units for managing power conversion. These units are installed on a 
support plate specifically designed which is installed in the forward cargo area of the aircraft.

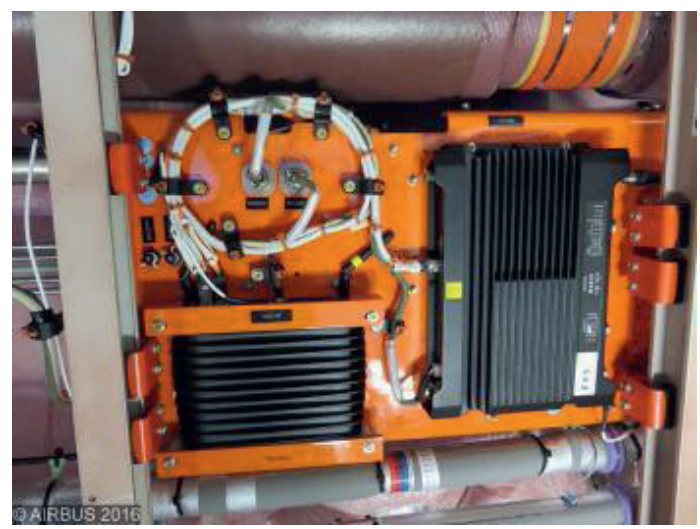

Fig. 3. Pre-equipped plate with connectivity system

All the CSTT data are sent over an Ethernet connection to the computing unit which records them. A part of these data is processed in realtime to allow automatic management of the different operational modes. It can therefore automatically detect the aircraft flight phases and act accordingly. In particular, by commanding the $4 \mathrm{G}$ router, the computing unit automatically activates (resp. deactivates) the $4 G$ communication when the aircraft is on ground (resp. in flight). In doing so, after each landing, a large set of data acquired during flight (approximately 5,000 parameters selected amongst the 24,000) is automatically downloaded from the aircraft to the ground. The $4 G$ link indeed allows sending the data directly from the aircraft to data lakes inside the Airbus network in a secured way over the standard telecommunication infrastructure.

An internal community of Airbus specialists can then access this high amount of data and proceed to various analyzes in very different technical fields.

\section{Challenges and success key factors for quick implementation}

One of the main challenges encountered was the time constraint. The target at start of the very beginning of the project was to install such a demonstrator on an in-service aircraft within a few months whereas traditionally, developing and certifying in-service aircraft equipment is generally a matter of years.

First, a quick development has been possible as it was built on existing components. Lab and flight tests were then also done in a short time frame. As for certification, it focused on safety aspect, ensuring that the system could be installed and operated on aircraft - for a limited period of time - in a safe way. Long-term reliability of the functional aspect was not deeply demonstrated (aircraft can fly safely at its full capability even if the Skywise demonstrator system cannot ensure its function).

The very special team spirit around this project has been a key factor for its success. Efficiency was achieved thanks to a reduced team - with an "agile" spirit - of people coming from many different Airbus domains: flight test instrumentation, engineering, information technologies, certification, design, manufacturing, customer support, safety, environmental and electrical qualification. All stakeholders were directly involved and strongly supported by internal sponsors. Direct communication channels were used to allow quick decision when needed.

Thanks to this, certification was reached 5 months after project start. It was installed on the first in-service aircraft one month later. Finally, to this date, 11 aircraft (5 different airlines over 4 continents) have flown with such a system since December 2016.

\section{Technical difficulties encountered}

Many technical challenges have been encountered during the project. Some of them are exposed hereafter.

One objective was to make the installation of the system as easy as possible, in particular to allow installation on already operating aircraft without generating service interruption. A preequipped plate and a harnesses kit were designed to this end. Thanks to this, and to the installation concept of CSTT (use of an available slot in the electronic bay), an installation over one to two nightshifts is generally possible thus allowing installation of the system directly in the airline facilities in its home base airport.

Another challenge was to ensure a $4 \mathrm{G}$ worldwide coverage. Many tests were done - in particular across borders - to secure automatic configuration of the $4 \mathrm{G}$ router so that connection can be established in any country. A special roaming contract was signed with a telecommunication operator to allow 4G (or $3 G$ as a fallback option) in every region of the world and in most countries.

Next technical issue relates to the management of data downloading to the ground. Whereas the nominal use case is to record data during flight and download all of it directly after landing, some technical risks were indeed to be anticipated. Typically, there could be poor communication (or even no communication at all) in some airports and/or during some time 
slots (e.g. 4G not deployed yet, overcrowded network). Therefore, the system needed to be robust against interruption and low download speeds. Algorithms were put in place to manage download interruptions without losing data. Additionally, the number of downloaded parameters has been adjusted (typically 5,000 parameters) to avoid backlog increase and internal storage in the on-board system was ensured to allow saving the data for approximately 1 month in case of difficulties.

\section{Results and benefits}

After 18 months of operations on 11 aircraft, more than 10 Terabytes of data have been collected.

Meetings have been held with engineering staff from airlines to target key topics and then propose analysis and algorithms using these data.

This led to a very good feedback from customers and concrete results. For example, the root cause of certain abnormal deteriorations could be quickly identified and appropriate recommendations given to the operator. It also led to a better understanding on how some avionics systems are used, and allowed proceeding to predictive maintenance on some components.

An additional benefit was the ability to know the real 4G heat map on more than 200 aircraft worldwide by monitoring and precisely correlating the aircraft geographical position, the signal level and type, and the resulting bitrate. Indeed, whereas $4 G$ seems to have become the standard nowadays, it is not yet reality everywhere or may have very different performance levels, making this type of information very valuable.

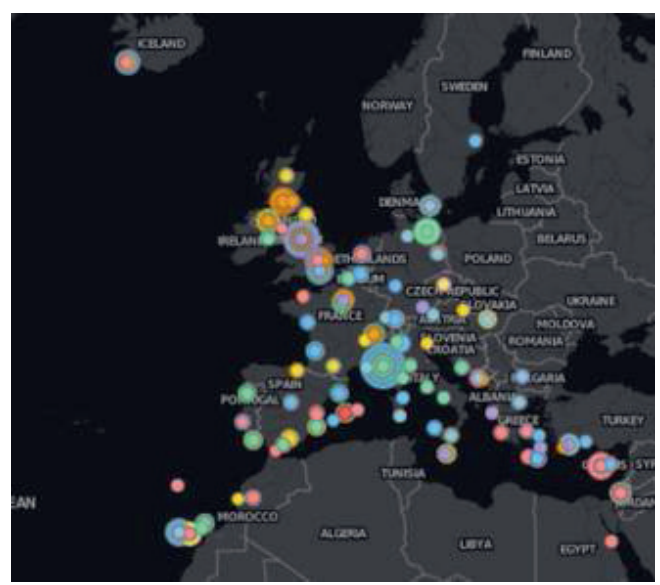

Fig. 4. Heat map for connectivity on European airports

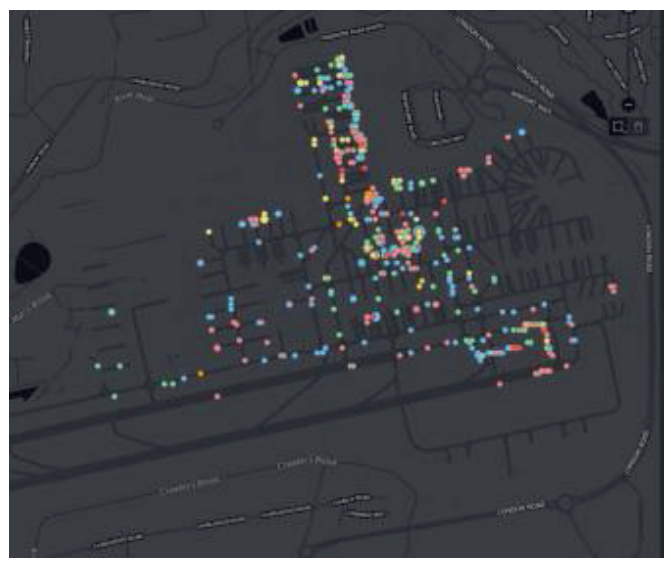

Fig. 5. Heat map for connectivity on an airport

\section{Conclusion}

The systems reached its targets by confirming the value of acquiring and analyzing more aircraft data, validating the strategic vision held by the Skywise project. It also allowed to de-risk the industrial solution and to work in partnership with a set of customers which expressed a very good feedback.

Consequently to this new "in-service demonstration" approach, the project has been internally awarded in 2018.

\section{Glossary}

COTS Commercial-of-the-shelf

CSTT Customer Support Test Tool

FTI Flight Test Instrumentation

PATS Production Aircraft Test System

SA Single Aisle

TB Terabyte(s) 